\title{
8. Über den Einfluß der Röntgenstrahlen auf das Einsetzen aer Glimmentladung ${ }^{1}$ ); \\ von Erich Marx.
}

\section{Einleitung.}

In meiner Abhandlung über die Geschwindigkeit der Röntgenstrahlen zeigte ich, daß der dort beschriebene Nullapparat im wesentlichen dadurch charakterisiert ist, daB im Falle eines von den Röntgenstrahlen negativ angetroffenen Potentials der schwingenden Elektrode von dem der Elektrode gegenüber befindlichen Elektrometer starke negative Ladungen angezeigt werden, während im Falle des Antreffens eines positiven Potentials die Elektrometerausschläge eine schwache positive Ladung anzeigten. Während diese schwachen Ausschläge aus der durch die Röntgenstrahlen hervorgerufenen Ionisation verständlich waren, reichte diese Erklärung für das Auftreten der starken negativen Ausschläge nicht aus. Die Größenordnung dieser Ausschläge war nur verständlich ${ }^{2}$ ), wenn man annahm, daB sie durch die Feldenergie der bestrahlten Elektrode bedingt sei, was sich auch dadurch offenbarte, daB die stärksten Ausschläge von einem, im Dunkeln wahrnehmbaren Leuchten des Gases begleitet waren. Das Charakteristikum der Versuchsanordnung bestand also darin, daB nur bei negativ angetroffener Elektrode eine wirkliche Feldentladung der Elektrode eintrat, bei positiver nicht. Das polare Einsetzen der selbständigen Entladung wurde von mir dadurch zu erklären versucht, daB die am $\mathrm{Pt}$ freiwerdenden, langsamen Elektronen den Potentialfall in bekannter Weise (vgl. nächstes Kap.) derart beeinflussen, daB die Bedingungen für das Einsetzen der Glimmentladung eintreten muBten. Dieses Einsetzen mubte hiernach ausbleiben, wenn die Kathode keine

1) Abdruck aus den Ber. d. k. Sächs. Ges. d. Wissenscl. 60. p. 248. (mit einigen Zusätzen und Ergänzungen).

2) E. Marx, Physik. Zeitschr. 6. p. 772. 1905; Verh. d. Deutsch. Phys. Ges. 10. p. 157. 1908. 
hinreichend langsame Kathodenstrahlung in genügender Menge abgab. Dies ist bei Al der Fall; am Al-Fenster entstehen, auch wenn es Kathode ist, langsame Kathodenstrahlen höchstens in ganz verschwindender Anzahl $^{1}$ ); es werden dort im wesentlichen nur Strahlen von großer Geschwindigkeit (Braggs Emergence-Radiation) frei, und zwar von dieser beträchtlich mehr an der Austrittsseite der Röntgenstrahlen, also am Al-Fenster, als beim Auftreffen auf die Elektrode.2)

In vorliegender Arbeit sollen die Verhältnisse, welche im Nullapparat der Geschwindigkeitsmessung dieses polare Einsetzen der Glimmentladung bedingen, gesondert untersucht werden. Dies erschien um so wünschenswerter, als in den bisherigen Versuchen, wie $\mathrm{Hr}$. W. Wien so freundlich war, mir brieflich mitzuteilen, ein experimenteller Nachweis für ein derartiges Verhalten der Glimmentladung nicht enthalten ist. Es existiert nämlich in der Tat von quantitativen Untersuchungen über den EinfluB der Röntgenstrahlen auf die Glimmentladung, soweit ich feststellen konnte, nur eine Würzburger Dissertation von Herweg. ${ }^{3}$ ) In dieser ist aber der Einflub der Röntgenstrahlen nur für den Fall untersucht, daB weder die Elektroden noch die Glaswand des Entladungsgefäßes, soweit sie von Einflub sein kann, von den Strahlen getroffen werden.

\section{Die Ausbildung der Glimmentladung.}

Bevor auf die eigenen Versuche und ihre Diskussion eingegangen wird, erscheint es zur Vereinfachung der Darstellung zweckmäBig, kurz an folgende Vorstellungen zu erinnern $)$, die sich auf Grund der Ionenhypothesen über das Zustandekommen der Glimmentladung herausgebildet haben: Durch das Anlegen einer höheren Spannung an die Elektroden werden an der Kathode Elektronen frei. Ist das Gefälle von hinreichender Größe, so entsteht in einiger Entfernung von der Kathode infolge von Ionisation durch StoB ein Gebiet gröBerer Leitfähigkeit. Die in diesem Bereich freiwerdenden positiven Ionen werden von der Kathode angezogen und erzeugen

1) W. Wien, Gött. Nachr. 1908.

2) W. H. Bragg, Trang of the Royal. Soc. of South Australia 32. 1908.

3) J. Herweg, Ann. d. Phys. 19. p. 333. 1906.

4) Vgl. J. J. Thomson, Elektr. Durehg, in Gasen p. 506. 1906. 
dort beim Auftreffen neue Elektronen, die ihrerseits wieder in einiger Entfernung von der Kathode die Leitfähigkeit er. höhen. Auf diese Weise nimmt der Potentialfall in einiger Entfernung von der Elektrode stark ab, und infolgedessen, da die Spannung an den Elektroden konstant ist, an den Elektroden selbst zu. In dem sich einstellenden stationären Zustand ist der Potentialfall im wesentlichen auf die Elektroden beschränkt; die Stellen maximaler Ionenproduktion zeigen die bekannten Leuchterscheinungen. An der Anode ist alsdann freie negative Elektrizität, an der Kathode freie positive Elektrizität im Gasraum vorhanden.

\section{Die Versuchsanordnung.}

Die Versuchsanordnung ist in Fig. 1 schematisch dargestellt. Eine Hochspannungsbatterie von der bekannten Feussner-Bornhäuser.Type von 500 Akkumulatoren führt über einen Amylalkoholwiderstand von etwa $40000 \mathrm{Ohm}$ zur Elektrode des EntladungsgefäBes. Der zweite Batteriepol wird geerdet. Ein Braunsches Elektrometer liegt direkt an der Elektrode. Die Variierung des anzulegenden Potentials erfolgt von der Batterie aus zunächst durch Kurbel-

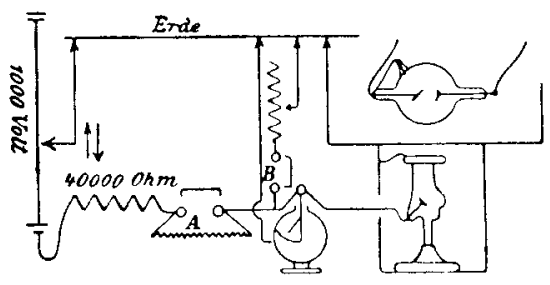

Fig. 1. schaltung. Hierbei ist darauf zu achten, daB die Aufladung der Elektrode und des elektrostatischen Voltmeters kontinuierlich und nicht stoßweiße erfolgt, weil es sonst nicht gelingt, größere Spannungen an die Elektrode anzulegen. Bei plötzlichem Einschalten lädt sich bekanntlich die Elektrode oszillatorisch auf, so daB die Momentanwerte der Spannung bis zu dem doppelten Wert des Batteriepotentials steigen können. ${ }^{1}$ ) Um ein langsames Aufladen zu ermöglichen, leistete mir ein alter Stromschlüssel $A$, dessen Ebonit etwas leitend geworden war, gute Dienste. Diese Leitfähigkeit ist durch Parallelzeichnen eines Widerstandes in der Fig. 1 angedeutet. Einige Sekunden nach Einstellungen der Batteriekurbel zeigt das Elektrometer bei offenem Schlüssel $A$

1) E. Warburg, Ann. d. Phys. 5. p. 818. 1901. 
annähernd das gleiche Potential wie bei Einschaltung. Um aber aber jede Überspannung beim Einschalten ganz sicher zu vermeiden, dazu diente der zweite Schlüssel $B$. Dieser wird kurz vor Einschaltung von $A$ geschlossen. Hierdurch legt man den Batteriepol über einen sehr großen variablen Widerstand zur Erde, so daß ein AbfluB erfolgt, der das Potential an der Stelle der Verzweigung etwas heruntersetzt, ohne dab die Spannung des Elektrometers wegen der GröBe des Widerstandes $A$ so schnell sinkt. Ist $A$ dann geschlossen, so kann der Widerstand $B$ durch eine Schraube bis zur Ausschaltung kontinuierlich gesteigert werden. (Ich benutzte einen Gummischlauch, der Amylalkohol enthielt, und dessen Querschnitt durch eine Klemme. verringert wurde.) Ist dann dieser Widerstand bis zur Ausschaltung vergröBert, so wird $B$ geöffnet, und falls keine Entladung erfolgt ist, wird bei offenem $B$ erst der Widerstand hinter $B$ wieder eingeschaltet, alsdann $A$ geöffnet, 100 Volt durch die Kurbel zugeschaltet, dann erst $B$ und nachher $A$ geschlossen. Auf diese Weise läBt sich sehr einfach das zunächst angelegte Potential kontinuierlich steigern und so das maximale Potential finden, das die Elektrode noch gerade aushält.

Bei dieser Untersuchung handelt es sich im übrigen nicht um die Auffindung ron Erscheinungen, die eine feinere Messung nötig machen, vielmehr lag mir nur daran, größere Effekte festzustellen. Die Ablesegenauigkeit am Voltmeter beträgt etwa 10 Volt. Ist die Entladung aus irgend einem Grunde übergegangen, so muB man einige Zeit warten, bevor man wieder Spannung anlegt, weil die Leitfähigkeit des Gases nicht gleich verschwindet; auch kann man sie durch Sättigungspotentiale unturhalb der Entladungspotentiale beseitigen.

Ganz wesentlich ist peinlichste Isolation aller zuführenden Teile (Bernstein), weil ein AbflieBen hoher Spannungen über schlechte Leiter leicht zu Gleitftunkchen AnlaB gibt, und dann aus obigem Grunde ein hohes Potential nicht anlegbar ist. Im allgemeinen kann man als Kriterium dafür, daB man das maximal mögliche Potential angelegt hat, daB also Isolation usw. in Ordnung ist, die Regel nehmen, daß sich alsdann der Elektrode, durch vorsichtiges Anlegen, ein Potential erteilen läBt, das etwa 40 Proz. größer ist als dasjenige, das bei plötzlichem Einschalten der Batterie das Entstehen der Glimmentladung verursacht. 
Der Kasten, der das Entladegefäß enthält, ist völlig geschlossen, und hat ein Aluminiumfenster, durch das die Röntgenstrahlen einer Röntgenrölìre von $5 \mathrm{~cm}$ Schlagweite fallen. An der VorderHäche des Kastens ist ein Drahtnetz in die Metallwand eingelötet, um beobachten zu können. Die ganze Schaltung ist von einem großen Zinkblech weit überragend überdacht. Der Einfluß der Röntgenstrablen wird natürlich nur so untersucht, daB erst dann die Zulassung der Röntgenstrahlen vorgenommen wird, wenn es sich zeigt, daß die Einschaltung der Röhre ohne Einfluß ist, solange das Al-Fenster des Kastens mit Blei bedeckt ist.

Evakuiert wurde mit einer Hg-Pumpe, bis zu solchem Druck, dab bei den angegebenen Röhrendimensionen und der verwendeten Spannung die Anode noch positives Licht zeigte, und der erste dunkle Kathodenraum etwa $1-2 \mathrm{~cm}$ betrug. Es kommt hierauf nicht genau an. Das Gas selbst muB natürlich trocken sein. Wenn die Röhren längere Zeit stehen oder $a b$. geschmolzen sind, wird das Glimmlicht immer weißlicher und ist nach etwa 8 Tagen völlig weiß. Das Vakuum verändert sich nicht merklich während Wochen. Es ist nicht ausgeschlossen, daB der als Kitt für die Fenster verwendete Marineleim den Gasinhalt verändert und daB das Al-Fenster zum Teil den Sauerstoff in den Luftresten bindet. Ist das Glimmlicht in dieser Weise verändert, so konnte ich die im folgenden beschriebenen Erscheinungen nicht erhalten. Die Intermittenz der Entladung, die nach Capstick ${ }^{1}$ ) in komplizierten Gasen eintritt, dürfte hiermit in Zusammenhang stehen, indem sie schon im Vorbereitungsstadium des Gases vor dem Eintritt der leuchtenden Entladung in Erscheinung tritt.

Die Al-Fenster bestanden aus Al-Folie von $4 / 100 \mathrm{~mm}$ Dicke und waren derartig zwischen zwei Glasringe mit Marineleim gekittet, daB die Al-Folie über den Rand des oberen Ringes herübergebörtelt war und durch eine Klemme Kontakt angebracht werden konnte. Die Fenster hatten ein Lumen von $3,7 \mathrm{~cm}$ und waren auf abgeschliffene Flansche, unter Zwischenkittung eines weiteren Glasringes, mit Marineleim so aufgekittet, daß sie gegen das Glas, elektroskopisch geprüf, isolierten. Die Versuche gelingen nur bei völliger Trockenheit des Glases.

1) Capstiek, Proc. Roy. Soc. 63. p. 356. 1898. 
Ist das Fenster nicht völlig isoliert, so können die freien Ladungen, welche die Polarisationsgebiete bilden, von den Glaswänden über das Fenster abflieBen. Eine geringe Leitfähigkeit zwischen Glas und Fenster genügt, um alsdann bedeutend höhere Potentiale an die Elektrode anlegen zu können, als bei völliger Isolation. Man hat deshalb hier das, auf den ersten Blick paradox erscheinende, Verhalten, da $\mathrm{B}$ bei geringer Überleitung zum Fenster das Entladungspotential der Glimmentladung viel höhere Werte hat, als bei peinlicher lsolation. Dies wird besonders hervortreten in dem Fall, daB der dunkle Raum, der ja isoliert, am Fenster liegt, während im anderen Fall der EinfluB geringer ist, da ja dann durch das Gas selbst eine Leitung hergestellt ist.

\section{Experimente bei nicht bestrahlten Flektroden.}

Es kamen zwei Entladungsröhren, die in Figg. 2 und 3 dargestellt sind, zur Verwendung. Als Elektroden dienen die Al-Folien, die in späteren Versuchen als Fenster benutzt werden.

Für die Röhre Fig. 2, bei der weder die Elektroden

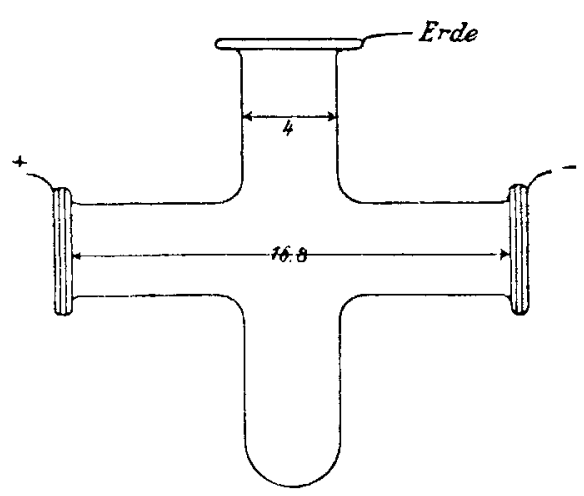

Fig. 2. noch die in Betracht kommende Glaswand ron Köntgenstrahlen getroffen wurde, ergab sich folgendes Resultat:

Tabelle I.

\begin{tabular}{c|c}
\hline $\begin{array}{c}\text { ohne Röntgen- } \\
\text { strahlen }\end{array}$ & $\begin{array}{c}\text { mit Röntgen- } \\
\text { strahlen }\end{array}$ \\
\hline \hline 740 & $\mathbf{7 2 0}$ \\
730 & 710 \\
740 & 710
\end{tabular}

Dieses Ergebnis ist in Übereinstimmung mit den Angaben von Herweg. Hr. Herweg fand in einem GefäB, in welchem die Glaswände nicht getroffen wurden, eine Erniedrigung der Entladespannung von 12-14 Volt bei $2 \mathrm{~mm}$ Druck und einer Entladespannung, die ohne Bestrahlung 620 Volt betrug. 
Wurde die Röhre Fig. 3 benutzt, so daB die Röntgenstrahlen zwar nicht die Elektroden, wohl aber die Glaswand trafen, so nahm die Erniedrigung der Entladespannung beträchtlich zu. Es wurde beobachtet:

Tabelle II.

\begin{tabular}{c|c}
\hline \hline $\begin{array}{c}\text { ohne Röntgen- } \\
\text { strahlen }\end{array}$ & $\begin{array}{c}\text { mit Röntgen- } \\
\text { strahlen }\end{array}$ \\
\hline \hline 770 & 700 \\
760 & 710 \\
780 & 710 \\
760 & 690
\end{tabular}

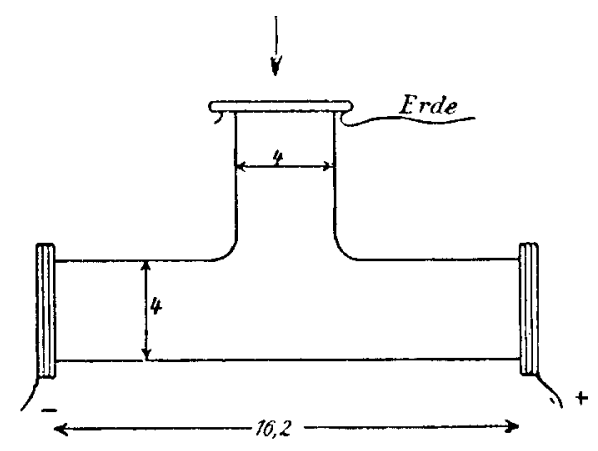

Fig. 3.

Die Erklärung für die Erniedrigung des Entladungspotentials in diesen beiden Versuchen ist nach den oben skizzierten Vorstellungen über das Zustandekommen der Glimm. entladung darin zu finden, daB durch die Röntgenbestrahlung die Leitfähigkeit des Gases in einiger Entfernung von den Elektroden so stark erhöht wird, dab an diesen Stellen der Potentialfall beträchtlich abnimmt. Da konstantes Potential an den Elektroden liegt, so muB an diesen, und zwar speziell an der Kathode, auf die es in erster Linie ankommt, das Gefälle steigen. Es wird also durch künstliche, lokale Steigerung der Leitfähigkeit zwischen den Elektroden die Ausbildung derjenigen Feldverteilung, die für das Einsetzen der Glimmentladung wesentlich ist, erleichtert. Da im Falle der Röhre Fig. 3 die Sekundärstrahlung des getroffenen Glases in gleicher Richtung wirkt, so wird der von Herweg beobachtete Effekt in der Fig. 3 noch verstärkt. Wurde die Leitfähigkeit nicht wesentlich zwischen den Elektroden gesteigert, indem die in Fig. 3 abgebildete Röhre so bestrahlt wurde, daB beide Elektroden von Röntgenstrahlen getroffen wurden, so treten ganz andere Verhältnisse auf.

Es sollen diese hier bei diesem Rohr nicht näher erörtert werden, weil hier eine Strömung zwischen Kasten und Fenster und die veränderte Verteilung der elektrischen Kraftlinien Komplikationen bedingt. Fs sei nur so viel bemerkt, daB, 
wenn die Leitfähigkeit eine relativ gleichmäBige Verstärkung im ganzen Entladungsraum infolge der Bestrahlung erleiden würde, so wäre keine Erniedrigung des Entladungspotentials, sondern das Gegenteil zu erwarten, da alsdann die Ausbildung des charakteristischen Potentialgefälles erschwert würde.

\section{Experimente bei bestrahiten Elektroden.}

Bei den nun folgenden Experimenten wurde stets so verfahren, daB das Fenster, durch das die Röntgenstrahlen auf die bestrahlte Elektrode fielen, auf dem gleichen Potential wie der Schutzkasten sich befand. Es waren stets entweder beide geerdet oder beide auf dem gleichen Potential und die Elektrode geordet, wodurch bei gleicher Richtung des Gefälles kein Unterschied der Resultate eintrat. In letzterem Falle wurde der Kasten natürlich isoliert aufgestellt. Es soi aber erwähnt, daB außer den in folgendem beschriebenen Versuchen eine große Zahl anderer angestellt wurde, bei denen die Verteilung der elektrischen Kraftlinien bei Vorzeichenwechsel der Elektroden, in Hinsicht auf die Umgebung, speziell auf den elektrostatischen Schutzkasten geändert wurde. Dann

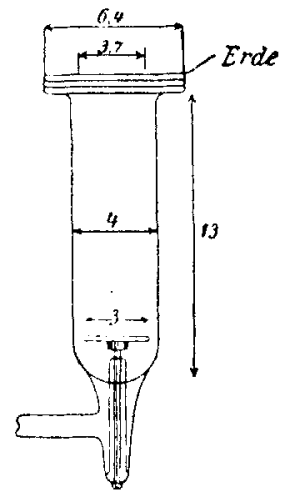

Fig. 4. treten merkwürdige, wesentlich kompliziertere Verhältuisse auf, auf die ich an dieser Stelle nicht eingehe, um die bereits hier nicht ganz einfach liegenden Verhältnisse nicht noch zu komplizieren. Es wurde bereits nachdrücklich betont, daß hier die örtlichen Ladungen, die sich am Glase bei der Bestrahlung ausbilden, sehr wesentlich beitragen zu dem Zustandekommen der hier beschriebenen Effekte, was ja auch leicht verständlich ist, wenn man bedenkt, daB hier (vgl. weiter unten) die Verteilung der freien Ladungen in der Nähe der Elektroden von aller wesentlichster $\mathrm{Be}$ deutung für die Ausbildung der Glimmentladung ist. Schon ein Erden des Glases in der Nähe der Elektrode kann bei nicht ganz trockenem Glase eine starke Verminderung des weiter unten beschriebenen Effèktes bei positiv angetroffener Elektrode zur Folge haben. Das gleiche ist, wie in Kap. III auseinandergesetzt, der Fall, wenn das 
Fenster nicht isoliert ist. Hier wurde stets so gearbeitet, daB ein äußeres Feld nicht vorbanden war, daB ferner die Kraftlinienverteilung konstant blieb, und auBerdem für peinliche Isolation und Trockenheit Sorge getragen wurde.

Es wurden zwei völlig gleiche Entladungsröhren hergestellt von der Form der Fig. 4. Bei der einen bestand die untere Elektrode aus $\mathrm{Al}$, bei der anderen aus $\mathrm{Pt}$.

Wurde an die Al-Elektrode einmal positives, das zweite Mal negatives Potential gelegt, so erhielt ich folgendes Resultat: Tabelle III.

$\mathrm{Al}_{(+)}$Fenster $_{\text {Erde }}$

\begin{tabular}{c|c}
\hline $\begin{array}{c}\text { mit Röntgen- } \\
\text { strahlen }\end{array}$ & $\begin{array}{c}\text { ohne Röntgen- } \\
\text { strahlen }\end{array}$ \\
\hline \hline 610 & 600 \\
600 & 600 \\
610 & 610
\end{tabular}

$\mathrm{Al}_{(-)}$Fenster $_{\text {Erde }}$

\begin{tabular}{c|c}
\hline \hline $\begin{array}{c}\text { mit Röntgen- } \\
\text { strahlen }\end{array}$ & $\begin{array}{c}\text { ohne Röntgen- } \\
\text { strahlen }\end{array}$ \\
\hline \hline $\mathbf{7 6 0}$ & $\mathbf{7 9 0}$ \\
$\mathbf{7 6 0}$ & $\mathbf{7 8 0}$ \\
$\mathbf{7 5 0}$ & $\mathbf{8 0 0}$
\end{tabular}

Es war also trotz der Symmetrie der Elektroden in Hinsicht des Materials ein schwacher aber sehr deutlicher EinfluB der Röntgenstrahlen zu beobachten, falls die untere Elektrode negativ getroffen wurde. Die Erklärung liegt, wie ich denke, zum Teil in den oben erwähnten Versuchen von Bragg und noch mehr in der durch die Konfiguration der Röhre zu den Elektroden bedingten Unsymmetrie der Bestrahlung; wir kommen später darauf zurück. Wurde in gleicher Weise die Röhre mit der Pt-Elektrode bestrahlt, so ergab sich folgendes Resultat:

Tabelle IV.

\begin{tabular}{|c|c|}
\hline \multicolumn{2}{|c|}{$\left.\mathrm{Pt}_{(t)^{1}}\right)^{1}$ Fenster $_{\text {Erd } \theta}$} \\
\hline $\begin{array}{l}\text { mit Röntgen- } \\
\text { strahlen }\end{array}$ & $\begin{array}{l}\text { ohne Röntgen- } \\
\text { strahlen }\end{array}$ \\
\hline $\begin{array}{l}600 \\
605 \\
600\end{array}$ & $\begin{array}{l}605 \\
600 \\
605\end{array}$ \\
\hline
\end{tabular}

\begin{tabular}{c|c}
\multicolumn{2}{c}{$\mathrm{Pt}_{(-)}$Fenster $_{\text {Erde }}$} \\
\hline \hline $\begin{array}{c}\text { mit Röntgen- } \\
\text { strahlen }\end{array}$ & $\begin{array}{c}\text { ohne Röntgen- } \\
\text { strahlen }\end{array}$ \\
\hline \hline 600 & 850 \\
610 & 880 \\
600 & 850
\end{tabular}

Hier also zeigte sich ein mächtiger EinfluB in dem Falle, daB die Pt-Elektrode negativ angetroffen wurde. Die Erklärung für die in diesen Tabellen wiedergegebenen Experimente ist

1) Vgl. die Anmerkung 2 auf p. 163. 
offenbar die schon bei früheren Beobachtungen unter ähnlichen Umständen von mir herangezogene. ${ }^{1}$ ) Betrachten wir zunächst den Fall, der in der vierten Tabelle rorliegt. Die vom $\mathrm{Pt}$ ausgehenden langsamen Kathodenstrahlen setzen in einiger Entfernung von der Elektrode durch die Erzengung einer beträchtlichen Leitfähigkeit das Gefälle herunter, dieses steigt an der Kathode und leitet so die Glimmentladung eher ein, als dies ohne Bestrahlung geschieht. Ist aber das bestrahlte Pt Anode, so werden die langsamen Kathodenstrahlen zurückgehalten, und können infolgedessen die Leitfähigkeit zwischen den Elektroden nicht erhöhen. In diesem Falle müBte aber trotzdem das gleiche Resultat der Röntgenstrahlen zu erwarten sein, wenn eine merklich langsame Kathodenstrablung von dem durchstrahlten Al-Fenster, das ja bei positiver Ladung der Pt Elektrode Kathode ist, ausginge. Dies ist aber nicht der Fall. Denn der Integraleffekt der Sekundärstrahlung ist bei auffallender Strahlung (Braggs Incidance Radiation) an sich sehr gering und der prozentische Anteil dieser geringen Strahlung an langsamen Strahlen wiederum, wie Hr. W. Wien ${ }^{2}$ ) zeigte, auBerordentlich geringer als bei Pt. Ebenso enthält die Austrittsstrahlung am Al-Fenster nur sehr wenig langsame Strablen. Die geringe Menge $\delta$-Strahlen, die am Al durch die Röntgenstrahlen frei wird, ist deshalb viel zu gering, um die Entladung rom Fenster aus zu erwirken. Das Verhalten der Entladung bei einer durch ein Al-Fenster bestrahlten Pt-Elektrode ist hiernach völlig verständlich. Nicht aber das Verhalten der nach Durchtritt durch das Al-Fenster getroffenen Al-Elektrode. Hier erscheint ein EinfluB des Potentials der Al-Elektrode zunächst aus Symmetriegründen unmöglich. Der Grund für das tatsächlich unsymmetrische Verhalten scheint mir nun hier in folgendem zu liegen: Die oben bereits erwähnten Braggschen Experimente erweisen für weiche $\gamma$-Strahlen einen Unterschied derjenigen Sekundärstrahlen, die beim Auftreffen der Röntgenstrahlen frei werden, gegenüber denen, die beim Durchtritt an der Kehrseite des bestrahlten Al entstehen. Bragg zeigte (für Röntgenstrahlen qualitativ Cooksey), daB an der Kehrseite des bestrahlten $\mathrm{Al}$ achtmal so viel $\beta$.Strahlen frei werden

1) E. Marx, Verh. d. Deutsch. Phys. Ges. 10. p. 148.1908.

2) W. Wi en, Nachr. d. Gött. Ges. d. Wissensch. 1908. 
als an der Auftreffseite. ${ }^{1}$ ) Es werden also am Al-Fenster sehr viel mehr schnelle Strahlen frei als an der Al-Elektrode. Dies gilt ceter. par.; hier, wo das Strahlenbündel nicht ausgeblendet ist, kommt hinzu, daB aus geometrischen Gründen die frei werdende Menge natürlich noch viel gröBer ist. Diese schnellen Strahlen treffen zum Teil die gegenüber befindliche Elektrode, zum Teil die Glaswand, und erzeugen überall, wo sie negative Ladung antreffen, $\delta$-Strablen. Es erscheint demnach durchaus möglich, daB bei völliger Symmetrie der bestrahlten Elektroden in Hinsicht des Materials, allein durch die Bestrahlungsrichtung polare Unterschiede auftreten: In der Tat lassen sich die Versuche der Tab. III für das bestrahlte Al so deuten, daB sich das bestrahlte $\mathrm{Al}$ so verhält, als wenn es langsame Kathodenstrahlen abgibt, während die durchstrablte Al-Folie keine langsamen Strahlen, sondern nur solche von so groBer Geschwindigkeit aussendet, daB keine partielle Beeinflussung des Gradienten zwischen den Elektroden eintritt. Das Fehlen einer merklichen $\delta$-Strahlung an der durchstrahlten Al-Folie ist also völlig hinreichend, um zu erklären, warum eine beträchtliche Herabsetzung des Entladepotentials nur eintritt, wenn die untere, bestrahlte Elektrode Kathode ist, nicht eintritt, wenn das Al-Fenster Kathode ist. - DaB dieser bei der bestrahlten Al-Elektrode diskutierte $\delta$-Strahleneffekt, der also nur indirekt den Röntgenstrahlen, direkt den durch letztere am Al-Fenster in groBer Menge erzeugten $\beta$-Strahlen zu verdanken ist, auch beim $\mathrm{Pt}$ mitwirkt, versteht sich natürlich von selbst. Bei den im folgenden untersuchten Röhren zeigte sich eine Beeinflussung des Entladungspotentials bei positiver Elektrode in umgekehrter Richtung wie die hier bei Bestrah. lung der Kathode festgestellte. Hierauf, und warum dieser Effekt sich hier nicht zeigte, darauf kommen wir später zurück. ${ }^{2}$ )

Wir haben uns mit diesem Experiment schon weitgehend dem Falle genähert, der in dem Nullapparat der Geschwindigkeitsmessung der Röntgenstrahlen vorliegt und der, wie in der

1) W. H. Bragg, I. e.

2) Anmerkung während der Korrektur. Bei Wiederholung der Versuche zeigte sich, daB das Al-Fenster nicht völlig isolierte. Nach Herstellung der Isolation ergaben sich auch bier die Zahlen, wie sie in Kap. V, SchluB, angegeben sin 1 . 
Einleitung hervorgehoben, hier gesondert untersucht werden sollte. Wird die Pt-Elektrode positiv angetroffen, so wird die Entladung nicht erleichtert, wird sie negativ angetroffen, so wird sie sehr bedeutend erleichtert, dann tritt die auslösende Wirkung der Röntgenstrahlung in Erscheinung. Dieses letzte Experiment zeigt, wie ich denke, daB meine Ausführungen ${ }^{\text {J }}$ über die Theorie des Nullapparates sich hier getrennt baben bestätigen lassen. $\mathrm{DaB}$ aber die Verhältnisse noch viel günstiger in polarer Hinsicht liegen, und der Erfolg meiner Messung noch einem weiteren, in gleicher Richtung wirkenden Effekte zuzuschreiben ist, das wird durch die folgenden Versuche klar, die sich noch weit mehr als der letzte Versuch, den Bedingungen im Nullapparat nähern.

Es ist nämlich im Falle des Nullapparates der Geschwindigkeitsmessung, der bestrahlten Elektrode parallel, und in etwa $3 \mathrm{~cm}$ Entfernung ein Faradayzylinder angebracht, der von den Röntgenstrahlen nicht getroffen wird. Die Hülle dieses Faradayzylinders ist geerdet. Die elektrischen Schwingungen, die von der bestrahlten Elektrode ausgehen, flieben über die Erdhülle des Zylinders zur Erde. Da die Frequenz der Schwingungen eine sehr groBe, der Abstand sehr gering ist, so stellt dio Elektrode, zusammen mit dem Faradayzylinder, einen Kondensator dar, welcher der abflieBenden Hertzschen Welle einen sehr geringen Widerstand bietet. Die Phasenverschiebung dieser Welle beim Durchtritt durch diesen Kondensator wird deshalb ebenfalls sehr gering sein, so daB sich die Welle nicht wesentlich anders beim Übertritt auf die geerdete Hülle verhalten wird, als wenn die Luftstrecke überbrückt wäre. Diese einfache Überlegung ergibt, daß das momentane Potential der geerdeten Hülle, das von der Hertzschen Welle herrührt, nur geringe Phasenverschiebung gegen das Potential der schwingenden Elektrode zeigen wird. Nur wird es infolge der Dämpfung und der Gröbe der metallischen Oberfläche des Faradayzylinders an diesem eine geringere Amplitude aufweisen, als die an der schwingenden Elektrode.

Will man demnach den Verhältnissen, die in diesem Apparate herrschen, gerecht werden, indem man Gleichstrom

1) E. Marx, Verh. d. Deutsch. Phys. Ges. 10. p. $138 \mathrm{ff} .1908$; ferner Ann. d. Phys. 28. p. 37. 1909. 
an Stelle des dort verwendeten Hochfrequenzstromes verwendet, so muB gegenüber der von den Röntgenstrablen getroffenen schiefgestellten Elektrode eine zweite Fläche angebracht werden, die selbst nicht von den Strahlen getroffen wird, und die ein, dem Vorzeichen nach gleiches aber wesentlich schwächeres Potential hat, als die bestrahlte Elektrode. Ich verwendete zunächst ein Entladungsgefä $B$ folgender Form, das gleichzeitig gestattet, auch unter diesen veränderten Bedingungen den kinfluß des Elektrodenmaterials zu untersuchen. Fensteröffnung und Röhrenlumen sind die gleichen, wie früher. Als Elektroden wurden zunächst schwach kalottenförmig gekrümmte verwendet, deren eine, die Pt-Elektrode, dieselbe war, die in

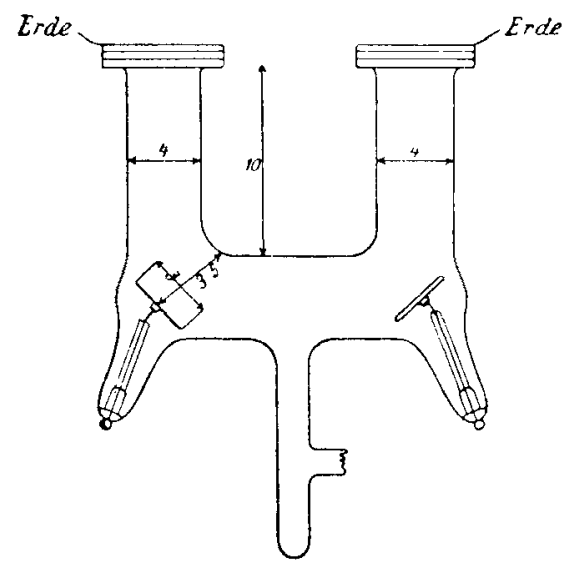

Fig. 5.

meinem Apparat zur Gesch windigkeitsmessung verwendet wurde. Die andere war eine Al-Elektrode von gleichen Dimensionen. Der Abstand der Elektroden usw. ist der Fig. 5 zu entnehmen. Legt man die eine dieser beiden Elektroden an die Hochspannung, so nimmt infolge der mangelnden Isolationsfähigkeit des Glases die andere sehr bald ein Potential von gleichem Vorzeichen an, das aber aus naheliegendem Grunde etwas schwächer als das der direkt metallisch anliegenden Elektrode ist. Dort, wo in den folgenden Tabellen der Index 0 an dem die Elektrode charakterisierenden Material angebracht ist, bedeutet dies, daB die betreffende Flektrode weder besonders geerdet, noch metallisch an eine Potentialquelle angelegt ist. 
Sie hat alsdann die durch die Leitung des Glases sich einstellende schwache Ladung der anderen Elektrode (Tabb. V und VII). In Tabb. VI und VIII sind die nicht bestrahlten Elektroden geerdet. Die beiden Fenster sind wiederum so durch die Marineleimkittung isoliert, daB sie gegen das Glas elektroskopisch geprüft isolieren.

\begin{tabular}{|c|c|c|c|}
\hline \multicolumn{4}{|c|}{$\mathrm{Pt}_{(-)}$Fenster $_{\text {Erde }}(\mathrm{Al})_{0}$} \\
\hline $\begin{array}{l}\text { mit Röntgen- } \\
\text { strahlen }\end{array}$ & $\begin{array}{l}\text { ohne Röntgen. } \\
\text { strahlen }\end{array}$ & $\begin{array}{l}\text { mit Röntgen- } \\
\text { strahlen }\end{array}$ & $\begin{array}{c}\text { ohne Röntgen- } \\
\text { strahlen }\end{array}$ \\
\hline 630 & 820 & 780 & 640 \\
\hline 625 & 800 & 770 & 650 \\
\hline 590 & 810 & 780 & 620 \\
\hline 590 & 800 & 760 & 630 \\
\hline 570 & 840 & 790 & 630 \\
\hline 590 & 820 & 780 & 680 \\
\hline \multicolumn{4}{|c|}{ Der Druck erhöht: } \\
\hline 700 & \multirow{2}{*}{$\begin{array}{ll}1080 \ldots & \text { (ev. höher, } \\
1000 \quad & \begin{array}{l}\text { Ende der } \\
\text { Batterie })\end{array}\end{array}$} & 890 & 810 \\
\hline 710 & & 880 & 790 \\
\hline 730 & 1050 & & \\
\hline \multicolumn{4}{|c|}{ Tabelle } \\
\hline \multicolumn{2}{|c|}{$\left.P t \_\begin{array}{l}\text { Al und } \\
\text { Fenster }\end{array}\right\}$ Erde } & \multicolumn{2}{|c|}{$\left.\mathrm{Pt}_{+} \begin{array}{l}\text { Al und } \\
\text { Fenster }\end{array}\right\}$ Erde } \\
\hline $\begin{array}{l}\text { mit Röntgen- } \\
\text { strablen }\end{array}$ & $\begin{array}{c}\text { ohne Röntgen. } \\
\text { strahlen }\end{array}$ & $\begin{array}{l}\text { mit Röntgen- } \\
\text { strahlen }\end{array}$ & $\begin{array}{c}\text { ohne Röntgen- } \\
\text { strahlen }\end{array}$ \\
\hline 510 & 680 & 720 & 560 \\
\hline 500 & 680 & 720 & 570 \\
\hline 520 & 690 & 680 & 580 \\
\hline 530 & 700 & 650 & 570 \\
\hline
\end{tabular}

Die Tabellen ergeben, daß bei negativ angetroffener Elek. trode eine Potentialerniedrigung von etwa 30 Proz. stattfindet. Die Potentialerniedrigung ist nicht sehr weitgehend konstant; es zeigt sich, daB eine Elektrode bei der ersten Bestrahlung schwerer anspricht, als wenn einmal eine Auslösung erfolgt ist. Oberflächenbeschaffenheit und Gasinhalt beeinflussen ihn. 
Der Effekt ist etwa von der gleichen Größe wie er vorhin bei Pt gefunden wurde. Ganz überraschend aber ist der Einfluß, der sich hier bei positiver Ladung der Pt-Elektrode zeigt. Das Experiment ist leicht zu demonstrieren, wenn allenthalben die oben angegebenen VorsichtsmaBregeln innegehalten werden ${ }^{1}$ ); es wirkt zunächst erstaunlich, dab man während der Bestrahlung ein höheres Potential an die Elektrode legen kann, als ohne Bestrahlung, dab also ein Abblenden der Röntgenstrahlen mit einem Bleiblech bei positiv angetroffener Elektrode gerade den umgekehrten Effekt ausübt, als im Falle der gewohnten negativen Auslösung. Bevor auf die Diskussion eingegangen wird, mögen die entsprechenden Resultate für Al mitgeteilt werden.

Tabelle VII.

Al bestrahlt.

\begin{tabular}{c|c}
\multicolumn{2}{c}{$\mathrm{Al}_{(-)}$Fenster $_{\text {Erde }} \mathrm{Pt}_{(0)}$} \\
\hline $\begin{array}{c}\text { mit Röntgen- } \\
\text { strablen }\end{array}$ & $\begin{array}{c}\text { ohne Röntgen- } \\
\text { gtrahlen }\end{array}$ \\
\hline \hline 575 & 725 \\
605 & 800 \\
620 & 810
\end{tabular}

\begin{tabular}{c|c}
\multicolumn{2}{c}{$\mathrm{Al}_{(+)}$Fenster $_{\text {Erde }} \mathrm{Pt}_{(0)}$} \\
\hline $\begin{array}{c}\text { mit Röntgen- } \\
\text { strablen }\end{array}$ & $\begin{array}{c}\text { ohne Röntgen- } \\
\text { strahlen }\end{array}$ \\
\hline \hline 740 & 720 \\
720 & 675 \\
760 & 720 \\
750 & 690 \\
740 & 680
\end{tabular}

Tabelle VIII.

\begin{tabular}{c|c}
\multicolumn{2}{c}{$\left.\mathrm{Al}_{-} \begin{array}{c}\text { Fenster } \\
\mathrm{Pt}\end{array}\right\}$ Erde } \\
\hline $\begin{array}{c}\text { mit Röntgen- } \\
\text { strahlen }\end{array}$ & $\begin{array}{c}\text { ohne Röntgen- } \\
\text { atrahlen }\end{array}$ \\
\hline \hline 485 & 503 \\
500 & 520
\end{tabular}

\begin{tabular}{|c|c|}
\hline $\begin{array}{l}\text { mit Röutgen- } \\
\text { strablen }\end{array}$ & $\begin{array}{l}\text { ohne Röntgen- } \\
\text { strablen }\end{array}$ \\
\hline 715 & 670 \\
\hline 710 & 650 \\
\hline 680 & 650 \\
\hline
\end{tabular}

Die hier angegebenen Zahlen ändern sich nicht, wenn anstatt der kalottenförmigen Elektroden, wie weiter unten angegeben, ebene genommen werden; es erscheint deshalb un-

1) Es wurde z. B. auf der Naturf.-Vers. in Köln demonstriert. Vgl. Physik. Zeitschr. 9. p. 731. 1908. 
nötig, besondere Tabellen hier anzugeben. Auch änderten sie sich nicht, wenn anstatt

$$
\left.\left.\mathrm{Al}_{-} \text {- } \begin{array}{c}
\text { Fenster } \\
\mathbf{P t}_{t}
\end{array}\right\} \text { Erde, } \mathrm{Al}_{\text {Erd } \theta}-\begin{array}{c}
\text { Fenster } \\
\mathrm{P}_{t}
\end{array}\right\}+
$$

genommen wurde.

In diesem Falle wurde aber, gemäB der Vorbemerkung zu Kap. $\nabla$ der Schutzkasten isoliert und mit dem Fenster gleichzeitig aufgeladen. Rein äuberlich möge bemerkt werden, daB ich bei ganz frischen Al-Elektroden die angegebenen Effekte, namentlich die bei negativ angetroffener Al-Elektrode, zum Teil nicht oder nur sehr schwach erhielt. Die Zahlen der Tabb. VII und VIII erhielt ich, als durch den Strom die $\mathrm{Al}$-Oberfläche bläulich angelaufen war. Auch beim Pt tritt etwas ähnliches ein, wenn auch nicht so ausgeprägt. Es zeigte sich immer, daB nach einiger Benutzung die frisch geschmirgelten, bzw. ausgeglühten Elektroden für die hier in Betracht kommenden Effekte geeigneter wurden. Ob hierbei die Gasabgabe der Elektroden oder direkt die OberHächenbeschaffenheit mabgebend ist, lasse ich dahingestellt. Für $\mathrm{Pt}$ sind die Auslösungseffekte etwa die gleichen, wie oben in Tab. IV. In Anbetracht der geringer getroffenen Oberfläche (die ja schief steht) sind sie aber gröBer. Vielmehr ist dies noch beim Al der Fall. Die Erklärung ist hier wohl ohne Zweifel darin $z u$ finden, dab die schnellen Strahlen, die bei parallelen Elektroden, wie oben in Tab. III wegen ihrer großen Weglänge unwirksam sind, hier zu einem Teil nicht geradeaus in Richtung der Stromlinien laufen, sondern auf die gegenüberliegende Glaswand treffen, dort machen sie $\delta$. Strahlen frei, die das schwache Gefälle, das sich in einiger Entfernung von der Kathode auszubilden beginnt, noch mehr schwächen, da sie dort infolge ihrer starken Absorption beträchtliche Leitfähigkeit erzeugen. Ist hierbei, wie in Tabb. V und VII, die zweite Elektrode, die unbestrahlt ist, nicht geerdet, so daB sie ebenfalls negatives Potential annimmt, so werden sämtliche an der Glaswand frei werdenden Strahlen in die Strombahn zum Fenster hin getrieben. Es ist demnach durchaus verständlich, daß in diesem Falle die Erniedrigung des Entladungspotentials viel beträchtlicher ist, als wenn die nicht bestrahlten Elektroden geerdet sind (VI und VIII). 
Es ist hiernach selbstverständlich, daB das gleiche dann eintreten wird, wenn die der Elektrode gegenüber befindliche Wand aus irgend einem anderen Material besteht, da ja an allen Materialien $\delta$-Strahlen frei werden, wenn Kathodenstrahlen sie treffen. In dem Nullapparat der Geschwindigkeitsmessung ist der Faradayzylinder parallel zu der schief stehenden bestrahlten Pt-Elektrode angebracht, dort treffen also die schnellen Strahlen, die am Pt frei werden, zum Teil auf die Hülle des Faradayzylinders und zum Teil auf die Glaswand. Die Zylinderhullle bildet mit der Elektrode einen Kondensator sehr geringer Reaktanz. Infolgedessen hat sie, wie hier die Glaswand, ein Potential von etwas anderer Größe, aber von im wesentlichen so geringer Phasenverschiebung, daB sie während der weitaus gröBten Zeit der Schwingung, gleiches Vorzeichen wie die Flektrode selbst hat. Die langsamen Elektronen, bzw. die $\delta$-Strahlen, werden deshalb nicht den gleichen Weg einschlagen, wie der Wechselstrom; vielmehr werden sie, falls die Elektrode stark negativ, der Faradayzylinder schwach negativ ist, sich im wesentlichen in Richtung der maximalen Potentialdifferenz, also zum Fenster hin bewegen. Die schnellen Elektronen aber treffen auf die Zylinderhülle und die Glaswand und machen dort $\delta$-Strahlen frei, die alsdann ihrerseits ihren Weg in der Richtung zum Fenster nehmen müssen, da sie durch das negative Feld der Elektrode und der Hülle abgestoBen werden. Auf die Verhältnisse bei positiver Phase wird gleich eingegangen werden.

Dab bei den oben angegebenen Versuchen der nicht geerdeten Elektrode keine wesentliche Bedeutung zukommt, wurde dadurch gezeigt, daB sie und das Fenster ihr gegenüber entfernt werden konnte, obne die

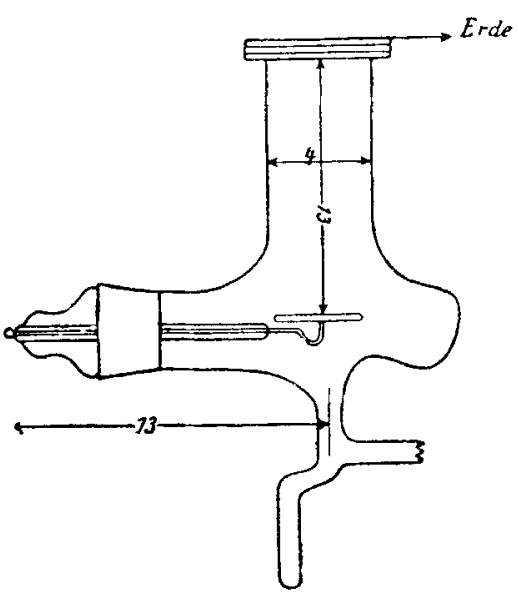

Fig. 6. angegebenen Zahlen merklich zu beeinflussen. Die freigelassene Elektrode hat ja nur das Potential des Glases. Auch das 
Ersetzen der Hohlelektroden durch ebene war ohne beträchtlicheren EinfluB. Es möge deshalb hier nur noch eine Zahlentabelle angeführt werden, die mit dem Glasrohr Fig. 6 angestellt ist. Hierbei ist die ebene Pt-Elektrode durch einen Schliff drehbar.

\section{$\mathrm{Pt}_{(+)}$Fenster $_{\text {Erdo }}$ \\ Horizontal}

\begin{tabular}{c|c}
\hline \hline $\begin{array}{c}\text { mit Röntgen- } \\
\text { strablen }\end{array}$ & $\begin{array}{c}\text { ohne Röntgen- } \\
\text { strahlen }\end{array}$ \\
\hline \hline 760 & 580 \\
750 & 570 \\
780 & 570
\end{tabular}

Tabelle IX.

Tabelle X.

\begin{tabular}{c|c}
\hline $\begin{array}{c}\text { mit Röntgen- } \\
\text { strahlen }\end{array}$ & $\begin{array}{c}\text { ohne Röntgen- } \\
\text { strahlen }\end{array}$ \\
\hline \hline 510 & 840 \\
520 & 830 \\
510 & 850
\end{tabular}

$\mathrm{Pt}_{(-)}$Fenster $_{\text {Erde }}$

Horizontal
$\mathrm{Pt}_{(+)}$Fenster $_{\text {Erde }}$

30 Arad Schiefstellung

\begin{tabular}{c|c}
\hline $\begin{array}{c}\text { mit Röntgen- } \\
\text { strahlen }\end{array}$ & $\begin{array}{c}\text { ohne Röntgen- } \\
\text { strahlen }\end{array}$ \\
\hline \hline 760 & 580 \\
760 & 560 \\
780 & 560
\end{tabular}

$\mathrm{Pt}_{(-)}$Fenster $_{\text {Erde }}$ $30 \mathrm{Grad}$ Sehiefstellung

\begin{tabular}{c|c}
\hline \hline $\begin{array}{c}\text { mit Röntgen- } \\
\text { strahlen }\end{array}$ & $\begin{array}{c}\text { ohne Röntgen- } \\
\text { strahlen }\end{array}$ \\
\hline \hline 520 & 890 \\
510 & 880 \\
520 & 890
\end{tabular}

Aus Tabb. IX und $\mathrm{X}$ ist zunächst ersichtlich, daB sowobl der Effekt bei positiv angetroffener Elektrode, wie der bei negativ angetroffener, mit Rücksicht auf die GröBe der angetroffenen Oberfläche, wesentlich stärker ist, wenn die Elektrode schräg, als wenn sie horizontal steht. Hierbei fällt auf, daB auch bei Horizontalstellung der positiven Elektrode eine beträchtliche Übergangserschwerung eintritt, während oben in Tab. IV kein EinfluB bei positiver Ladung und Horizontalstellung beobachtet werden konnte. $\mathrm{Da}$ der in die Augen springendste Unterschied zwischen Fig. 4 und Fig. 6 darin liegt, daB im ersten Falle die Glaswände die Elektrode ganz dicht umschließen, im zweiten aber beträchtlicher Spielraum zwischen Elektrode und Glaswand ist, so wurden zunächst die Bedingungen der Fig. 4 dadurch zu reproduzieren versucht, daB in eine Röhre vom Charakter der Fig. 6 ein Glaszylinder 
so hineingebracht wurde, daB die Elektrode von ihm eng um. schlossen wurde. Das Resultat war, daB hierdurch der positive Effekt um etwa 30 Proz. verringert wurde, also doch immerhin noch so deutlich bestehen blieb, daB die Diskrepanz nur zum Teil dem fehlenden Zwischenraum zwischen Glaswand und Elektrode zugeschrieben werden kann. Es ist demnach das wahrscheinlichste, daB hier gewisse Oberflächenbeschaffenheiten in dem Rohr Fig. 4 sich geltend machten ${ }^{1}$ ), die allein in diesem Falle ein Inerscheinungtreten des positiven Effektes verhinderten, da sonst in sämtlichen Versuchen diese Erscheinung beobachtet wurde. Auf diesen positiven Effekt, der hier völlig unerwartet in die Erschoinung trat, wird in folgendem näher eingegangen.

\section{Die Frhöhung des Fntladungspotentials bei positiv angetroffener Elektrode.}

Aus den angeführten Experimenten ergibt sich, daB unter dem Einfluß der Röntgenstrahlen solche Veränderungen an einer Elektrode, welche in positiver Ladung angetroffen wird, eintreten, daB das Einsetzen der Entladung beträchtlich erschwert wird. Nun hängt nach obigen Ausführungen über das Zustandekommen der Glimmentladung diese in erster Linie daron $a b$, dab der Kathodenfall eine bestimmte untere Grenze erreicht; wird das Erreichen dieser Grenze verhindert, so tritt keine Glimmentladung ein. Dieses Herabdrücken des maß. geblichen Kathodengefälles kann nun bei konstantem, anliegendem Potential seinen Grund darin haben, daß an einer anderen Stelle der Strombahn eine Erhöhung des Gefälles eintritt. Eine solche Erhöhung wird allgemein an den Elektroden dann eintreten, wenn die Polarisationsgebiete (vgl. Kap. II), welche sich um die Elektroden bilden, anwachsen. Wie man nämlich aus der Gestalt des Gradienten vor Eintritt der Glimm. entladung ersieht, ist in der Nähe der Anode freie negative Elektrizität vorhanden. Ein Steigern dieser freien negativen Elektrizität an der Anode hat ein Anwachsen des Anodenfalles zur Folge. Diese Steigerung muB dann eintreten, wenn Röntgen-

1) DaB dies der Fall war, ist in der Anmerkung 2 auf p. 163 ausgeführt. 
strahlen auf die Elektrode fallen, welche ein positives Potential von einigen hundert Volt hat. Denn die Kathodenstrahlen, welche an der bestrahlten Elektrode frei werden, bestehen nicht nur aus den schnellen Dornschen ( $\beta$-Strahlen) und den ganz langsamen Strahlen, sondern auch aus solchen Strablen, die einer Geschwindigkeit entsprechen, die durch einige hundert Volt derart beeinfluBt werden, daB sie auf kurzen freien Wegstrecken existenzfähig sind. Ihre Geschwindigkeit reicht nicht mehr zur Ionisation durch StoB aus, so daB sie an den Gasmolekülen hängen bleiben; es entstehen negative Ionen und Elektronionen. Durch diese $\mathrm{muB}$ die negative Volumladung an der Anode erhöht werden, es muB also der Anodenfall steigen, was gleichzeitig, da die Klemmenspannung konstant ist, ein Sinken des Kathodenfalles zur Folge haben muB. Dann aber muB sich eine Erschwerung des Einsetzens der Glimmentladung einstellen. Man wird also bei positiv angetroffener Elektrode eine größere Potentialdifferenz während der Bestrahlung anlegen können, als ohne Bestrahlung.

Jeder Vorgang, welcher die negative Volumladung an der Elektrode erhöht, wird in gleicher Richtung wie hier die direkt ankommenden Röntgenstrahlen wirken. Wenn demnach die Bedingungen derart sind, daß die Gesamtheit der bei Röntgenbestrahlung frei werdenden Kathodenstrablen, also nicht nur die langsamen, sondern durch irgend einen Zwischenvorgang, auch die schnellen, freie negative Elektrizität an die Anode schaffen können, so wird man erwarten mulssen, daB der Erschwerungseffekt zunimmt. Dies ist aber der Fall, wenn die Elektrode schräg gestellt ist, und ihr gegenüber sich eine Wand befindet, die ein Potential hat, das nur wenig geringer ist, als das der Elektrode. Dann treffen nämlich alle Kathodenstrahlen, auch die schnellen, die durch die Röntgenstrahlen an der Elektrode frei werden, auf die Wand. Dort erzeugen sie $\delta$-Strahlen, die sich nun in dem schwachen Felde zwischen Anode und Wand zur Anode bewegen, und zum Teil in der Nähe der Anode die freie negative Volumladung erhöhen. Hiernach ist also die beträchtliche Erhöhung der Entladeerschwerung bei Schiefstellung der Anode, der Mitwirkung der schnellen $\beta$-Strahlung zu danken, die an der bestrablten Elektrode selbst frei wird, und die bei Horizontalstellung für die 
Anreicherung von negativer Elektrizität an der Anode wegen der GröBe der freien Weglänge nicht in Betracht kommt.

In dem Nullapparat der Geschwindigkeitsmessung müssen infolge der oben wiederholt diskutierten analogen Verhältnisse gleiche Vorgänge in Erscheinung treten. Nur sind dort wegen des höheren Druckes und höheren Potentials die charakteristischen Teile der Entladung räumlich viel mehr konzentriert. Es muB auch hier bei angetroffener +-Elektrode die Entladung erschwert, bei angetroffener -.Elektrode erleichtert werden. Hierzu kommen noch zwei weitere Umstände. Erstens ein beträchtlicher Entladeverzug bei +-Elektrode. Es zeigt sich nämlich, daß die Ausbildung des Glimmlichtes, im Falle daB die Pt-Elektrode positiv ist, nicht sofort erfolgt, sondern daß benachbart zu dem Potential, das sich noch dauernd anlegen läBt, sich ein Gebiet befindet, innerhalb dessen das Potential für kurze Zeit ohne Eintritt der Entladung anlegbar ist. Bei negativem $\mathrm{Pt}$ ist ein solches Gebiet, das einen merklichen hier nach Sekunden zählenden Entladeverzug zeigt, von mir nicht beobachtet worden. Ferner aber hat in diesem Apparat das Al-Fenster eine viel kleinere Oberfläche als die Pt-Elektrode, Die Folge davon ist, daB die Entladung schwerer einsetzte, wenn das Fenster Kathode ist, als wenn es Anode ist. In der Tat kann man etwa das doppelte Potential im ersten Falle anlegen als im zweiten. Für die Verhältnisse im Nullapparat kommt der, hier zuerst beobachtete, positive Erschwerungseffekt nicht direkt in Betracht. Denn dort wird das Potential so reguliert, daB ohne Bestrahlung eine Entladung ïberhaupt nicht stattfindet. Für die Funktion dieses Apparates wäre hinreichend, daB keine Erleichterung der Feldentladung bei positiv angetroffener Elektrode statthat, was allein bisher in der Theorie des Apparates von mir zu erklären versucht wurde. $\mathrm{DaB}$ aber sogar eine Erschwerung stattfindet, war nicht vorausgesehen, ist aber für die $\mathrm{Zu}$ verlässigkeit des Funktionierens sicherlich ein nicht unbeträchtlicher Sicherheitsfaktor. Auch die Verzögerungserscheinung, d. b. die beträchtliche Zeit, die vergeht, bevor nach Abblenden der Röntgenstrahlen die Entladung bei positiver Elektrode einsetzt, ist der Polarität des Entladungseffektes im Nullapparat durchaus günstig: Denn der Anstieg des Anodenfalles 
ist bedingt durch negative Ionen, die sich in der Umgebung der Anode bilden. Erst nachdem dieses negative Polarisationsgebiet an der Anode verschwunden ist, steigt der Kathodenfall. Der Versuch zeigt, daß dies längere Zeit dauert nach Abblenden der Strahlen. - Es wird also im Nullapparat, im Falle Antreffens der positiven Phase, dieses Polarisationsgebiet, wenn es sich gebildet hat, auch in der folgenden negativen, unbestrahlten Phase fortbestehen. Dort wirkt es aber derart, daB der Kathodenfall heruntergeht und geschwächt wird, da ja das für die Glimmentladung hinreichende Gefälle ein positives Polarisationsgebiet an der Kathode fordert. Das negative Gefälle wird also dadurch noch mehr geschwächt, und da es bereits ohne diese Schwächung nicht zur Glimmentladung ausreichte, so wird jetzt a fortiori in der unbestrahlten Phase keine Entladung erfolgen können.

Die mehrfach beschriebene Tatsache, daB bei richtiger Anordnung meines Apparates nur bei den größten negativen Potentialamplituden eine selbständige Feldentladung bei der Bestrablung einsetzt, nicht aber bei positiv angetroffenen, ist, wie ich denke hier für sich, getrennt vom Apparat, als notwendig bewiesen. Diesen Beweis und die hieraus sich ergebende wesentliche Stütze für die Richtigkeit meiner Auffassung von den Vorgängen im Apparat zur Geschwindigheitsmessung der Röntgenstrahlen, möchte ich als wesentliches Resultat vorliegender Untersuchung betrachten. Wie ich bereits in der in dieser Zeitschrift ${ }^{1}$ ) mitgeteilten Theorie des Apparates ausführte, ist mir jetzt kein experimentelles Ergebnis des Geschwindigkeitsapparates mehr bekannt, das nicht durch experimentelle Ergebnisse unter vereinfachten Bedingungen reproduziert werden kann, und das nicht in direkter Konsequenz des Bildes liegt, das in der Theorie enthalten ist und das die Vorgänge im Apparate, so weit ich sehe, vollständig beherrscht.

Leipzig, Physik. Institut, Ende Juli 1908.

1) E. Marx, Ann. d. Phys. 28. p. 37. 1909.

(Eingegangen 27. Oktober 1908.) 